\title{
Lung-Protective Ventilation for SAH Patients: Are These Measures Truly Protective?
}

\author{
Gregory Kapinos • Astha Chichra
}

Published online: 11 September 2014

(c) Springer Science+Business Media New York 2014

Acute lung injury and acute respiratory distress syndrome (ALI/ARDS) are common in patients with critical neurological illnesses and herald a worse outcome. It has been reported in 15-40\% of patients suffering from an aneurysmal subarachnoid hemorrhage (SAH) [1, 2]. Any type of acute brain injury can trigger ALI/ARDS, but even more so, the systemic inflammatory response syndrome (SIRS) seen in the acute and delayed phases of SAH exposes these patients to this type of catastrophic respiratory deterioration [3]. Furthermore, hemodynamic augmentation has been the mainstay of treatment for vasospasm/delayed cerebral ischemia in patients with SAH and the timing of the initial therapy as well as the utilization of each component (hypertension, hypervolemia, and inotropic enhancement) varies widely [4]. Hypervolemia, stressed cardiac function, and early recourse to blood transfusion can easily lead to pulmonary edema with worse outcomes. But it is easy to understand that in order for the injured and actively ischemic brain to receive adequate oxygen, gas exchange in the lungs must occur optimally [3]. Finally, because nearly a third of mortality after SAH is due to medical and not neurological complications [5, 6], early implementation of strategies to prevent, even partially, the development of ALI/ARDS is primordial.

\section{G. Kapinos ( $\square)$}

Departments of Neurosurgery \& Neurology, Hofstra North Shore-LIJ School of Medicine, North Shore-LIJ Health System, 300 Community Drive, Tower, 9th floor, Manhasset, NY 11030, USA

e-mail: kapigreg@gmail.com

\section{A. Chichra}

Division of Pulmonary and Critical Care, Department of Medicine, Hofstra North Shore-LIJ School of Medicine, Manhasset, NY, USA
The pathophysiology of ALI/ARDS is complex, and a "double hit" model has been proposed [7]. Severe brain injury results in the "first hit" with an adrenergic surge and systemic inflammation, making the lung more susceptible to injury $[3,7]$. The "second hit" results from non-neurological variables such as infections, transfusions, and mechanical ventilation (MV) [3, 7]. One of the major goals of care in such patients should include optimization of oxygen delivery to prevent hypoxemia and, hence, secondary neurological insults.

Lung-protective ventilator settings (LPS) have been proposed as a cornerstone in not only the management of but also in the prevention of ALI/ARDS, while injurious ventilator settings (IVS) definitely facilitate the appearance of this lung failure [8,9]. In this issue of the journal, Marhong and colleagues [10] report a single-center retrospective experience of ventilator practices in a mixed intensive care unit (ICU) in adult SAH patients. The authors describe the incidence of ALI/ARDS as well as the adherence to LPS. They included only patients who required MV within $72 \mathrm{~h}$ of their admission. Authors [10] define LPS as tidal volume (TV) $\leq 8 \mathrm{~mL} / \mathrm{kg}$ of predicted body weight, positive end-expiratory pressure (PEEP) $\geq$ $5 \mathrm{~cm} \mathrm{H}_{2} \mathrm{O}$, and peak or plateau pressure $\leq 30 \mathrm{~cm} \mathrm{H}_{2} \mathrm{O}$. The development of ARDS was defined by $\mathrm{PaO}_{2} / \mathrm{FiO}_{2} \leq 300$ along with new bilateral lung opacities on chest X-ray within a day of hypoxemia.

Currently accepted thresholds for lung-protective ventilation (LPS) $[8,9]$ were not met in approximately onethird of patient days, suggesting that this is a target for quality improvement. But initial MV settings were not associated with the subsequent development of ARDS. Furthermore, Marhong et al. [10] found no correlation between one, two, or all three components of LPS, or even IVS and the subsequent occurrence of ARDS. Readers 
should appreciate the detailed analysis [10] into not only dichotomized endpoints and parameters, but also severity of ARDS, adherence to one, two, or three lung-protective criteria and time for the development of ARDS. In this cohort [10], only the number of previously validated [8,9] ARDS risk factors (sepsis, shock, aspiration, pneumonia, and transfusion) was independently associated with the subsequent development of ARDS.

Although the authors [10] mention that the Berlin definition was used to define their criteria for ARDS, they could not exclude other causes of pulmonary edema due to the retrospective study design. Hence, implications of the results may be limited due to a misclassification of volume overload and other causes of pulmonary edema as ARDS.

According to Claassen's physiologic derangement score (PDS) [5, 6], an alveolar-arteriolar gradient $>125 \mathrm{~mm} \mathrm{Hg}$ gives the patient a score of at least 3 , which implies a 3 -month mortality of at least $30 \%$. In-hospital mortality from ARDS in SAH can even be higher, according to our recent national in-patient survey [1]. This cohort [10] averaged an in-hospital mortality of $35 \%$, an ICU length of stay of 6 days, and MV period of 3 days. The fact that pressure-control and volume-control modes represented only $22 \%$ of the studied patients is also representative of usual ventilation techniques used in North American centers treating SAH gravely ill patients. Half of patients were between 6 and $8 \mathrm{~mL} / \mathrm{kg}$, which also seem to be the reflective of common practices, and only $58 \%$ had all three LPS criteria met, because pressure support is commonly used, with patients raising their TV on their own. The cohort included $6 \%$ of patients "exposed" to very high TV, giving also some interesting data to help support or refute the hypothesis that IVS is indeed harmful and conducive to ARDS.

The overall demographics, morbidities, and patterns of medical care that are described in this study [10] are matching the average SAH population usually treated in modern countries by specialists publishing large cohorts [1-6], and this sampled SAH population is grossly similar to the results of our U.S. national in-patient survey of practices [1]. This suggests good representation for fair extrapolation and generalizability of conclusions.

While a significant portion of these SAH patients underwent MV within the acute phase, and in spite of ARDS affecting half of these patients, with lenient definitional criteria, the majority of the subjects were actually breathing spontaneously on MV and, therefore, generating TV above what is considered LPS. This raises questions regarding the differential effects of high TV seen during spontaneous breathing versus exposure during positive pressure ventilation, as rightly discussed by the authors [10]. While pressures and TV generated during spontaneous breathing modes might be categorized as high, they may not be necessarily injurious. Perhaps patients with spontaneous breathing ability and relatively adequate oxygenation should not be sedated in order to impose tight pressure-control ventilation aiming at strict "adherence" to LPS. Contrary to the evidence strongly supporting that applying LPS prevents ALI/ARDS and IVS enhances the occurrence of ALI/ARDS [8, 9], this study [10] may be only suggesting that patients with spontaneous breathing, not adhering to LPS, do not have a higher incidence of ARDS. But readers of this article should be cautious in concluding that initial LPS are not justified for all mechanically ventilated SAH patients, solely based on the fact that ARDS seemed to occur independently of initial MV settings and that mortality was unchanged in this study. Actually, the increase in ICU and hospital mortality with the occurrence of ARDS was also found in this small study not to be statistically significant, as compared to the non-ARDS group [10], which is contrary to the evidence for the ominous prognostic significance of ARDS [1-3, 59]. This may mean that these patients were not so sick, after all, breathing spontaneously and sample size may have been too small to detect further independent associations. In addition, it remains common sense to set initially the MV to the least assistance and to minimize the risk of volutrauma, anyway. PEEP $\geq 5 \mathrm{~cm} \mathrm{H}_{2} 0$ not only addresses avoidance of alveolar collapse, which is involved in the development of ARDS, but is also used as a baseline standard setting to avoid basal band atelectases, commonly seen in ventilated SAH patients confined to decubitus with poor mobilization.

The current study should, therefore, be seen as a platform for future prospective multicenter studies, designed to identify the best-suited MV strategy, tailored to our fragile SAH patients. Based on the current level of evidence for LPS from large studies for prevention of ARDS [8, 9], it is our opinion that lung-protective ventilation should still be used as an initial MV strategy in critically ill patients with neurological illnesses. Furthermore, in patients with SAH and at least one ARDS risk factor, it still seems judicious, according to this study [10], to apply LPS. Finally, in case of confirmed ARDS, LPS should still be used in SAH patients, even to the level of reaching permissive hypercapnia, before going to airway pressure release ventilation. Indeed, original concerns about intracranial hypertension with these two latter MV settings can be mitigated by recent data [11, 12] supporting standard escalation in ARDS treatment even in the brain-injured patient.

Acknowledgments The authors are grateful to Effie Singas, MD, FACP, FCCP for her thoughtful input in the revision of the manuscript.

Disclosures Dr. Kapinos and Dr. Chichra report no relevant disclosure. 


\section{References}

1. Veeravagu A, Chen YR, Ludwig C, Rincon F, Maltenfort M, Jallo J, Choudhri O, Steinberg GK, Ratliff JK. Acute lung injury in patients with subarachnoid hemorrhage: a nationwide inpatient sample study. World Neurosurg. 2014;82(1-2):e235-41.

2. Kahn JM, Caldwell EC, Deem S, Newell DW, Heckbert SR, Rubenfeld GD. Acute lung injury in patients with subarachnoid hemorrhage: incidence, risk factors, and outcome. Crit Care Med. 2006;34(1):196-202.

3. Stevens RD, Lazaridis C, Chalela JA. The role of mechanical ventilation in acute brain injury. Neurol Clin. 2008;26(2):543-63.

4. Meyer R, Deem S, Yanez ND, Souter M, Lam A, Treggiari MM. Current practices of triple-H prophylaxis and therapy in patients with subarachnoid hemorrhage. Neurocrit Care. 2011;14(1): 24-36.

5. Wartenberg KE, Mayer SA. Medical complications after subarachnoid hemorrhage. Neurosurg Clin N Am. 2010;21(2): 325-38.

6. Claassen J, Vu A, Kreiter KT, Kowalski RG, Du EY, Ostapkovich N, Fitzsimmons BF, Connolly ES, Mayer SA. Effect of acute physiologic derangements on outcome after subarachnoid hemorrhage. Crit Care Med. 2004;32(3):832-8.

7. Mascia L. Acute lung injury in patients with severe brain injury: a double hit model. Neurocrit Care. 2009;11(3):417-26.
8. Determann RM, Royakkers A, Wolthuis EK, Vlaar AP, Choi G, Paulus F, Hofstra JJ, de Graaff MJ, Korevaar JC, Schultz MJ. Ventilation with lower tidal volumes as compared with conventional tidal volumes for patients without acute lung injury: a preventive randomized controlled trial. Crit Care. 2010;14(1):R1.

9. Serpa Neto A, Cardoso SO, Manetta JA, Pereira VG, Esposito DC, Pasqualucci Mde O, Damasceno MC, Schultz MJ. Association between use of lung protective ventilation with lower tidal volumes and clinical outcomes among patients without acute respiratory distress syndrome: a meta-analysis. JAMA. 2012;308(16): 1651-9.

10. Marhong JD, Ferguson ND, Singh JM. Ventilation Practices in Subarachnoid Hemorrhage: A Cohort Study Exploring the Use of Lung Protective Ventilation. Neurocrit Care. 2014. doi:10.1007/ s12028-014-0014-8.

11. Petridis AK, Doukas A, Kienke S, Maslehaty H, Mahvash M, Barth H, Mehdorn HM. The effect of lung-protective permissive hypercapnia in intracerebral pressure in patients with subarachnoid haemorrhage and ARDS. A retrospective study. Acta Neurochir (Wien). 2010;152(12):2143-5.

12. Marik PE, Young A, Sibole S, Levitov A. The effect of APRV ventilation on ICP and cerebral hemodynamics. Neurocrit Care. 2012;17(2):219-23. 